\title{
Steady-state responses in the somatosensory system interact with endogenous beta activity
}

1 Michel J. Wälti ${ }^{1,2 *}$, Marc Bächinger ${ }^{1,2}$, Kathy L. Ruddy ${ }^{1,3}$, Nicole Wenderoth ${ }^{1,2}$

$2{ }^{1}$ Neural Control of Movement Lab, Department of Health Sciences and Technology, ETH Zurich,

3 Switzerland

$4 \quad{ }^{2}$ Neuroscience Center Zurich (ZNZ), University and ETH Zurich, Zurich, Switzerland

$5 \quad{ }^{3}$ Institute of Neuroscience, Trinity College Dublin, Ireland

6 * Correspondence:

7 Michel J. Wälti

8 michel.waelti@hest.ethz.ch

9 Keywords: steady-state evoked potentials, entrainment, somatosensory system, neural

10 oscillations, vibrotactile stimulation

\section{Conflict of Interest}

12 The authors declare that the research was conducted in the absence of any commercial or financial

13 relationships that could be construed as a potential conflict of interest.

\section{Author Contributions}

15 MJW, MB and KLR designed research; MJW performed research; MJW and MB analyzed data; MJW, $16 \mathrm{MB}, \mathrm{KLR}$ and NW wrote the paper.

\section{Funding}

18 This research was funded by the Swiss National Science Foundation (No. 320030_175616).

\section{Acknowledgments}

20 We thank Pearl Saldanha and Marionna Münger for their help with data acquisition, and the Statistical

21 Counseling Service of ETH Zurich, especially Janine Burren, for methodological support. We also 22 acknowledge the support of the Neuroscience Center Zurich (ZNZ). 


\section{Abstract}

25 Brain oscillations have been related to many aspects of human behavior. To understand a potential causal relationship, it is of great importance to develop methods for modulating ongoing neural activity. It has been shown that external rhythmic stimulation leads to an oscillatory brain response that follows the temporal structure of the presented stimulus and is assumed to reflect the synchronization of ongoing neural oscillations with the stimulation rhythm. This interaction between individual brain activity and so called steady-state evoked potentials (SSEPs) is the fundamental requirement of neural entrainment. Here, we investigate whether neural responses to rhythmic vibrotactile stimulation, measured with EEG, are dependent on ongoing individual brain oscillations, and therefore reflect entrained oscillatory activity. For this, we measured phase synchronization in response to rhythmic stimulation across various frequencies in the alpha and beta band. Three different stimulation intensities were applied for each frequency relative to the individual sensory threshold. We found that a higher stimulation intensity, compared to lower intensities, resulted in a more pronounced phase synchronization with the stimulation signal. Moreover, EEG responses to low stimulation frequencies closer to individual beta peak frequencies revealed a higher degree of entrainment, compared to stimulation conditions with frequencies that were more distant to endogenous oscillations. Our findings provide evidence that the efficacy of vibrotactile rhythmic beta stimulation to evoke a SSEPs is dependent on ongoing brain oscillations.

\section{Introduction}

44 During cognitive tasks brain rhythms of different frequencies can be recorded at the scalp using electroencephalography (EEG), that are correlated with cognitive and behavioral processes. In recent years, it has been suggested that various non-invasive methods, such as repetitive transcranial magnetic stimulation (rTMS) or transcranial alternating current stimulation (tACS) are capable of frequencyspecific modulation of brain rhythms. Hence, they have been used to test the causal relationship between the strength of neuronal oscillations and behavior (for a review, see Thut, Schyns, \& Gross, 2011). However, the interaction of these externally applied magnetic or electric fields with ongoing brain rhythms is still poorly understood. An alternative form of frequency-specific modulation of brain rhythms can be achieved with rhythmic sensory stimulation which induces steady-state evoked 
usually measured with electroencephalography (EEG) and have been documented in the visual (steadystate visually evoked potentials, SSVEPs), the auditory (auditory steady-state responses, ASSRs) and the somatosensory (steady-state somatosensory evoked potentials, SSSEPs) systems (for a review, see Vialatte, Maurice, Dauwels, \& Cichocki, 2010). However, the underlying mechanism of SSEPs, is still debated (see Zoefel, Ten Oever, \& Sack, 2018). While some studies report evidence for entrainment of ongoing brain oscillations by showing an interaction between stimulation and endogenous activity (Notbohm, Kurths, \& Herrmann, 2016; Schwab et al., 2006), others found responses to be nothing more than regular repetitions of evoked neural potentials (Capilla, Pazo-Alvarez, Darriba, Campo, \& Gross, 2011; C. Keitel, Quigley, \& Ruhnau, 2014). In contrast to simply overlaying evoked potentials, entrainment modulates brain oscillations. To test whether brain responses to rhythmic sensory stimulation reflect entrainment, it is therefore necessary to provide evidence of a dependency on ongoing brain oscillations. This dependency arises from theoretical considerations and has been the main research interest in studies supporting the entrainment hypothesis (see Zoefel et al., 2018). The so-called Arnold tongue describes a classical model of entrainment between two oscillators depending on stimulation rhythm and intensity (Pikovsky, Rosenblum, Zaks, \& Kurths, 1999). The model predicts that a high stimulation intensity evokes a larger response than a low intensity but also entrainment to be most pronounced when the stimulation frequency matches the frequency of the endogenous system (e.g. Fröhlich, 2015). In a recent study, Notbohm et al. (2016) reported that the coupling of EEG oscillations with rhythmic visual flicker follows the characteristics of the Arnold tongue. They showed that the EEG response to the stimulation depends on both the stimulation intensity and the distance between the flicker frequency and the participants' individual alpha frequency (IAF; Notbohm et al., 2016). Other studies found similar effects for tACS over occipital cortex which either increases EEG power at participants' visual alpha frequency (Helfrich et al., 2014; Zaehle, Rach, \& Herrmann, 2010), or biases ongoing alpha oscillations in visual cortex towards the exogenous stimulation frequency (Minami \& Amano, 2017). Most previous studies have tested entrainment in visual cortex targeting the alpha rhythm. Here we test whether it is also possible to entrain endogenous brain rhythms of primary sensorimotor cortex via rhythmic tactile stimuli. It is well known that the sensorimotor cortex exhibits strong activity in the alpha $(8-12 \mathrm{~Hz})$ and beta band $(15-30 \mathrm{~Hz})$ when subjects are at rest (Crone et al., 1998; Fransen, Dimitriadis, van Ede, \& Maris, 2016). During sensorimotor processing and, particularly, during motor execution, both rhythms desynchronize (Bauer, Oostenveld, Peeters, \& Fries, 2006; Kilavik, Zaepffel, Brovelli, MacKay, \& Riehle, 2013; Spitzer \& Haegens, 2017) but resynchronize quickly once the movement is completed, implicating both rhythms in sensorimotor processing (Kilavik et al., 2013; Pfurtscheller \& Lopes da Silva, 1999; Ploner, Gross, Timmermann, 
Pollok, \& Schnitzler, 2006; Rajagovindan \& Ding, 2011). Here we ask whether the alpha and the beta rhythms in primary sensorimotor cortex are equally susceptible to entrainment via tactile stimuli. We hypothesized that repetitive tactile stimulation at or close to the intrinsic alpha and beta frequency peaks of the sensorimotor system should be more effective than stimulation at other frequencies. To that end, we probed whether characteristics of the Arnold tongue can be found when measuring phase synchronization in response to vibrotactile stimulation at different frequencies in the alpha and beta bands. Three different stimulation intensities were applied for each frequency which were defined relative to individual sensory threshold. We expected steady-state EEG responses to be more pronounced for (i) high-intensity stimulations compared to low intensities, and (ii) at stimulation frequencies closer to individual alpha (IAF) and/or beta frequencies (IBF) compared to frequencies more distant from endogenous oscillations.

\section{Materials and methods}

\section{Participants}

10125 participants took part in the experiment in exchange for monetary compensation (20 Swiss francs

102 per hour). The participants had no reports of psychiatric disorders and were all right-handed according 103 to the Edinburgh Handedness Inventory (Oldfield, 1971). The study protocol was approved by the local 104 ethics committee and was conducted in accordance with the Declaration of Helsinki. All participants 105 provided written consent. 3 participants were excluded from the analysis due to technical problems 106 during EEG recording or because they did not follow the instructions (e.g. falling asleep during the 107 EEG recording), resulting in a final sample of 22 participants (female: 8; age: range $=18-29, \mathrm{M} \pm$ $108 \mathrm{SD}=22.8 \pm 2.6)$.

\section{Design and procedure}

110 The experiment was conducted in two parts. In a first part, we determined participants' IAF and IBF 111 in a simple finger tapping task. The second part consisted of the main experiment with different 112 conditions of vibrotactile stimulation to participants' right index finger. Throughout both parts, EEG 113 was recorded (see below).

\section{Part I: Detection of IAF and IBF}


115 Participants were comfortably seated in a dark sound-attenuated room, approximately $100 \mathrm{~cm}$ away

116 from a computer screen (27-inch) and were instructed to execute a self-paced finger tapping task with

117 their right hand, in which they tapped and gently squeezed their thumb against each of the other fingers

118 successively. One run consisted of an alternating green and red fixation cross appearing in the center

119 of the screen (each 9 times for 10 seconds). Green indicated that the participant should execute the

120 finger tapping, and red indicated that they should rest their hand. Each participant performed two runs.

\section{Part II: Vibrotactile stimulation}

122 During the main experiment, participants' right index finger was stimulated with a rhythmic

123 vibrotactile stimulation. For the stimulation, an earphone (YVE-01B-03, Yeil Electronics Co., South

124 Korea) with a diameter of $17 \mathrm{~mm}$ was taped to the participants finger. The earphone was modified to

125 produce vibrations via a coil actuator with a mass attached which vibrates when current is passed

126 through. The stimulation signals were generated in MATLAB (The MathWorks Inc., USA) and sent

127 to the stimulation devices via a National Instruments Card (NI-USB 6343) and an amplifier (Dancer

128 Design, St. Helens, UK). The stimulation was elicited by driving the stimulator with a carrier frequency

129 of $200 \mathrm{~Hz}$ which was frequency-modulated by a square wave corresponding to the stimulation

130 frequency (Breitwieser, Kaiser, Neuper, \& Müller-Putz, 2012). This time course of the driving voltage

131 had a resolution of $0.1 \mathrm{~ms}$. For each participant, the individual stimulation threshold was determined

132 prior to the main experiment by applying a $20 \mathrm{~Hz}$ stimulation stimulus which started at sub-threshold

133 intensity and increased in steps of $10 \%$ increments of output voltage until it was perceived. This

134 procedure was repeated three times. The mean values of the detection thresholds indicated the

135 individual stimulation threshold for each participant. Based on the individual stimulation threshold

136 three stimulation intensities were set: (i) sub-threshold intensity corresponding to $0.5 \mathrm{x}$ threshold

137 intensity (sub-threshold), (ii) a low supra-threshold intensity corresponding to $20 \mathrm{x}$ threshold intensity

138 (low), and (iii) a high supra-threshold intensity corresponding to $200 \mathrm{x}$ threshold intensity (high).

139 During the main experiment (Figure 1), all stimulation frequencies (i.e. $6-24 \mathrm{~Hz}$ ) were applied with

140 the same sub-threshold, low and high intensities, respectively. Note that the range of our experimental

141 frequencies was rather narrow such that all activate the same tactile mechanoreceptors (Meissner's

142 corpuscles, $5-50 \mathrm{~Hz}$; see Johansson \& Flanagan, 2009). After threshold detection, the main

143 experiment began which consisted of 3 blocks each consisting of 10 stimulation conditions which were

144 carried out in random order (resulting in 30 stimulation conditions in total). These 30 conditions were

145 composed of 10 different stimulation frequencies ranging from 6 to $24 \mathrm{~Hz}$ in steps of $2 \mathrm{~Hz}$, each

146 administered with the three stimulation intensities (sub-threshold, low or high). Each stimulation lasted 
147 for 40 seconds and was preceded by a 10 second period without stimulation. Participants were 148 instructed to sit as still as possible and direct their gaze at a white fixation cross centered on a black 149 screen.

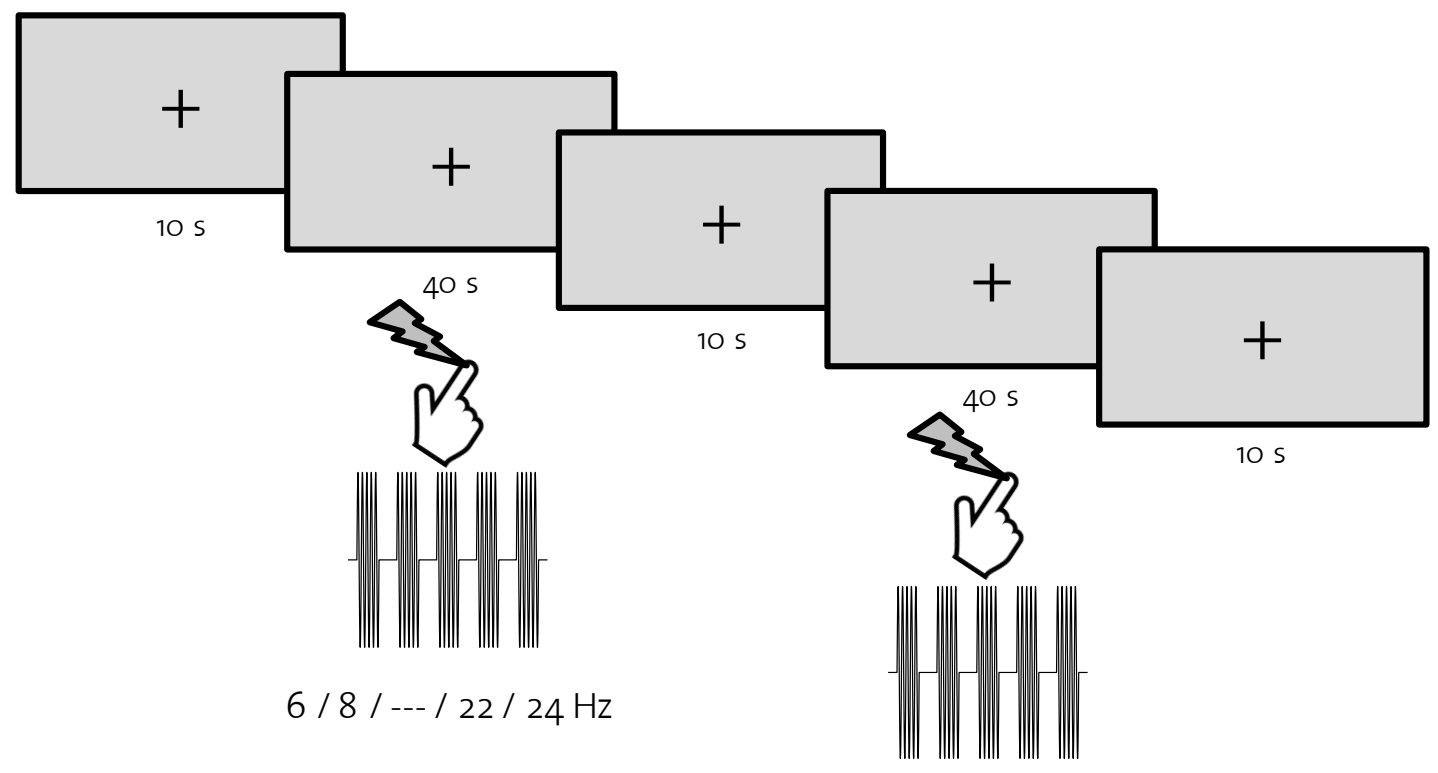

151 Figure 1. Experimental procedure. Participants' right index finger was stimulated with frequencies ranging 152 from 6 to $24 \mathrm{~Hz}$ at 3 different stimulation intensities. Each stimulation lasted for $40 \mathrm{~s}$, preceded by a $10 \mathrm{~s}$ period without stimulation (rest).

\section{Data acquisition}

155 EEG data were acquired at $1000 \mathrm{~Hz}$ using a 64-channel Hydrocel Geodesic EEG System (Electrical

156 Geodesic Inc., USA), referenced to $\mathrm{Cz}$ (vertex), with an online Notch filter $(50 \mathrm{~Hz})$ and high-pass filter 157 at $0.3 \mathrm{~Hz}$. Impedances were kept below $50 \mathrm{k} \Omega$.

\section{Preprocessing of EEG data}

159 EEG data for both parts of the experiment were preprocessed and analyzed offline. Data were cleaned 160 (detection and interpolation of bad electrodes), band-pass filtered $(5-25 \mathrm{~Hz})$ and further processed 161 using independent component analysis (ICA). Artifact components (ICs) were automatically detected 162 and removed from the data using a custom built toolbox (see Liu, Ganzetti, Wenderoth, \& Mantini, 163 2018).

\section{EEG data analysis}


166 To detect IAF and IBF, preprocessed data were epoched regarding the two conditions (movement and 167 rest; each from $+1 \mathrm{~s}$ to $+9 \mathrm{~s}$ after start of the condition) and analyzed using fast Fourier transform (FFT).

168 For each subject, frequency-band specific power differences in the alpha $(8-12 \mathrm{~Hz})$ and beta band (15 $169-25 \mathrm{~Hz}$ ) were calculated between movement and rest to detect event-related de-synchronization (ERD) 170 that typically appears during movement as well as event-related synchronization (ERS) immediately 171 after movement. Power changes were determined as percentage change from rest versus movement 172 (Pfurtscheller \& Lopes da Silva, 1999):

$$
\Delta \text { Power }=\frac{\text { power }(\text { rest })-\text { power }(\text { movement })}{\text { power }(\text { movement })} * 100 .
$$

174 The electrode(s) exhibiting the strongest $\Delta$ Power over the contralateral hemisphere (right hand, left 175 hemisphere) for the alpha and beta band, respectively, was selected and we determined (i) IAF as the 176 frequency with the biggest power increase between 6 and $14 \mathrm{~Hz}$ (range of the stimulation frequencies); 177 and (ii) IBF as the frequency with the most pronounced power increase between 16 and $24 \mathrm{~Hz}$. Similar 178 to before, we defined power increases as percentage change in power in the rest condition compared 179 to the move condition ( $\Delta$ Power) and normalized the resulting power spectrum to the power at the alpha 180 or beta peak respectively.

\section{Part II: Steady-state evoked potentials}

182 In order to evaluate general effects of vibrotactile stimulation on EEG signals, we measured frequency183 specific power and phase-based consistency of EEG time-series during stimulation. For this, 184 preprocessed EEG data from the main experiment were epoched to each stimulation condition $(+1 \mathrm{~s}$ to $185+39$ s relative to stimulation onset). Further, time-series data were cropped in 1 s segments without overlap which were later treated as separate trials. In order to analyze steady-state responses to the 187 stimulation frequencies we pre-defined a region of interest (ROI) consisting of nine electrodes over the 188 somatosensory area. For each participant, we evaluated the electrode that showed the strongest SSEPs across alpha $(6 \mathrm{~Hz}, 8 \mathrm{~Hz}, 10 \mathrm{~Hz}, 12 \mathrm{~Hz}, 14 \mathrm{~Hz})$ and beta $(16 \mathrm{~Hz}, 18 \mathrm{~Hz}, 20 \mathrm{~Hz}, 22 \mathrm{~Hz}, 24 \mathrm{~Hz})$

190 stimulation conditions separately. For this, data averaged across trials of each high-intensity 191 stimulation condition were transformed into its frequency components using a fast Fourier transform 192 (FFT) and power at the corresponding stimulation frequency as well as neighboring frequencies $(-2$ $193 \mathrm{~Hz},-1 \mathrm{~Hz},+1 \mathrm{~Hz},+2 \mathrm{~Hz}$ ) were determined for each electrode within the ROI. We then calculated 
194 percentage increase of power at the stimulation frequency compared to power at neighboring

195 frequencies for each electrode. To evaluate the electrode showing the strongest response across the

196 alpha stimulation conditions, we averaged the calculated power changes across the corresponding

197 conditions. The same procedure was carried out for the beta stimulation conditions. To determine

198 stimulation effects on frequency-specific power, FFT was performed on data that had been

199 preprocessed and averaged across trials, derived from these electrodes (strongest alpha and beta for

200 each participant). To determine frequency-specific phase consistency of the EEG signals, we further

201 calculated intertrial phase clustering (ITPC), which measures the extent of phase angle distributions at

202 each time-frequency point across trials (Cohen, 2014).

203 Part II: Phase-based synchronization between EEG and stimulation signal (entrainment)

204 To determine entrainment, we used data from the electrodes which were earlier defined as detecting 205 the strongest alpha and beta signals. We then measured changes in intersite phase clustering (ISPC)

206 between the preprocessed and averaged time-series data of each stimulation condition and the 207 corresponding stimulation signal. ISPC is a measure of phase-based connectivity between two time208 series signals (Cohen, 2014). Similar to ITPC, ISPC measures phase angle distributions, with the key 209 distinction that phase angle differences between two signals are measured. Similarly, to the EEG data, 210 the stimulation signals were band-pass filtered in the range from 5 to $25 \mathrm{~Hz}$ and down-sampled to 1000 $211 \mathrm{~Hz}$. The single-channel EEG time-series were further band-pass filtered in the range of $\pm 2 \mathrm{~Hz}$ regarding

212 the corresponding stimulation frequency. To detect changes in phase-locked activity in response to the

213 stimulation, we compared ISPC between the stimulation signal and the EEG signal during stimulation

214 with ISPC prior to each condition (i.e. preceding rest period). Similar to the previously described

215 procedure, EEG data prior to stimulation onset ( $-9 \mathrm{~s}$ to $-1 \mathrm{~s})$ was cropped in $1 \mathrm{~s}$ segments and then

216 averaged across trials. $\triangle$ ISPC was calculated by subtracting the mean pre-stimulation ISPC from the

217 mean ISPC during stimulation for each of the 30 conditions. All scripts to execute the experiment and

218 to preprocess and analyze EEG data were written in MATLAB (The MathWorks Inc., USA), and used

219 functions from EEGLAB (Delorme \& Makeig, 2004). Scripts using FFT, ITPC and ISPC analysis were

220 adapted from M. X. Cohen (Cohen, 2014). For the experiment presentation code, PsychToolbox was

221 used (Brainard, 1997).

\section{Statistical analysis}

223 All statistical analyzes were performed separately for conditions targeting alpha oscillations 224 (stimulation frequencies: $6 \mathrm{~Hz}, 8 \mathrm{~Hz}, 10 \mathrm{~Hz}, 12 \mathrm{~Hz}, 14 \mathrm{~Hz}$ ) and beta oscillations (stimulation 
225 frequencies: $16 \mathrm{~Hz}, 18 \mathrm{~Hz}, 20 \mathrm{~Hz}, 22 \mathrm{~Hz}, 24 \mathrm{~Hz}$ ). General effects of the stimulation signals on

226 frequency-specific power and ITPC were analyzed using repeated-measures ANOVA models in SPSS

227 Version 25 (IBM, USA). To test the hypothesized effects regarding phase-based connectivity between

228 stimulation and EEG signals, we used R (R Core Team, 2018) and the lme4 package (Bates, Mächler,

229 Bolker, \& Walker, 2015) to perform linear mixed effects analyzes. We used linear mixed effects

230 models because they account for variance arising from individual differences (Baayen, Davidson, \&

231 Bates, 2008). To ensure that observed effects are not driven by individual variability, subjects were

232 included in the models as random factors. Fixed effects represent the influence of experimental factors

233 on the dependent variable (here: $\triangle \mathrm{ISPC}$ ). In our models, the fixed effects include stimulation intensity

234 (sub-threshold intensity as baseline) and distance of the stimulation frequency to individual

235 endogenous frequencies in the alpha $(\Delta \mathrm{IAF})$, or the beta $(\Delta \mathrm{IBF})$ band. Validation of our models was

236 done by plotting residuals against fitted values.

\section{Results}

239 The current study was designed to reveal variations in the effect of vibrotactile stimulation on the 240 individual neuronal response in sensorimotor cortex. Participants were rhythmically stimulated at their 241 right index finger (dominant hand) using three stimulation intensities and ten stimulation frequencies 242 across the alpha and beta bands. Each stimulation lasted for 40 seconds and was preceded by a 10 243 second period without any stimulation.

\section{Detection of IAF and IBF}

245 To detect IAF and IBF, frequency-band specific power changes due to event-related de/synchronization (ERD/ERS) in a simple finger tapping task were determined for each subject in the alpha and beta bands. Figure 2 A/B show topographical plots and power spectra from an example subject, revealing strong power increases in the left primary motor areas (rest vs. movement) and clear peaks in the power spectra in both alpha and beta bands. Across subjects, average IAF was $11.0 \mathrm{~Hz}( \pm$ 2.1) and average IBF was $20.3 \mathrm{~Hz}( \pm 1.9$ ) (see Figures $2 \mathrm{C} / \mathrm{D}$ ). To evaluate whether the two spectral peaks in alpha and beta present two independent rhythms, we calculated linear correlation with the

252 Pearson correlation coefficient between IAFs and IBFs across subjects. We found only a weak negative 253 correlation $(\mathrm{r}=-0.22, \mathrm{p}=0.319)$ and conclude that the power changes related to the finger tapping task 254 found in alpha and beta represent two distinct endogenous brain rhythms rather than harmonics. 

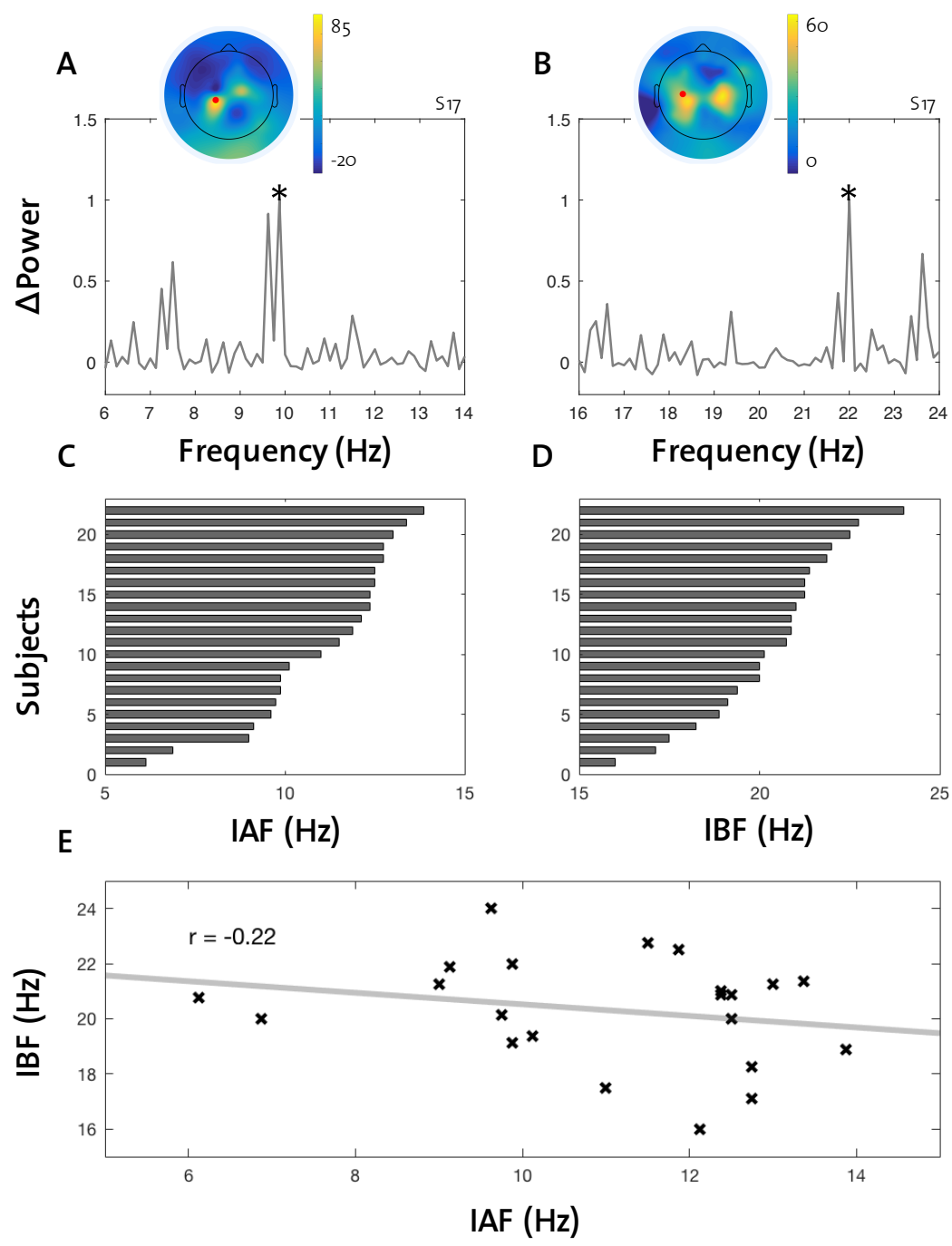

256 Figure 2. Participants' IAF and IBF. Detection of participants' endogenous peak frequencies in the alpha (IAF) and beta (IBF) bands by calculating frequency-band specific power changes due to event-related de/synchronization (ERD/ERS) in a simple finger tapping task. A: Example subject's topographical plot of $\Delta$ Power in the alpha band and power spectrum of the electrode showing the highest value (red dot). IAF was determined as frequency with the highest $\Delta$ Power (here: $9.875 \mathrm{~Hz}$, marked with an asterisk). B: Same example subject's topographical plot and power spectrum as in A, but for the beta band (IBF: $22 \mathrm{~Hz}$, marked with an asterisk). C: Ordered IAF frequencies across all subjects. D: Ordered IBF frequencies across all subjects. E: Depiction of correlation with regression line between IAF and IBF values. Pearson correlation coefficient reveals only a weak negative linear dependency $(r=-0.22)$.

\section{Stimulation intensity differences on steady-state evoked potentials in alpha and beta}

In order to evaluate entrainment effects, we first need to determine whether our stimulation protocol reveals typical characteristics of steady-state evoked potentials over the somatosensory area across 
268 alpha and beta frequencies. Further, we are interested in general differences between the two frequency 269 bands regarding effects of stimulation intensity. Therefore, a two-way repeated measures ANOVA 270 with factors frequency band (alpha and beta) and stimulation intensity (sub-threshold, low, high) was 271 used to detect general effects of different stimulation conditions on frequency-specific power and 272 phase-clustering (ITPC) values. Figures 3 and 4 depict stimulation effects averaged across all subjects.

273 Figure 3 shows changes in the power spectra across all stimulation conditions and Figure 4 reveals 274 effects on ITPC. Our first analysis used power as dependent variable (derived from FFT). The results 275 from the ANOVA reveal a main effect of stimulation intensity $(F(2,20)=50.7, p<0.001)$, but not of 276 frequency band $(\mathrm{F}(1,21)=0.7, \mathrm{p}<0.411)$. Bonferroni adjusted post-hoc comparisons between 277 intensities revealed a significant difference between all three levels (sub-threshold vs. low: $p=0.030$; 278 sub-threshold vs. high: $\mathrm{p}<0.001$; low vs. high: $\mathrm{p}<0.001)$. Further, the interaction between frequency 279 band and stimulation intensity show a significant effect, meaning that vibrotactile stimulations in alpha 280 and beta have different effects on frequency-specific power across the different intensities $(\mathrm{F}(2,20)=$ $2813.7, p=0.041)$. This steeper increase in power across the stimulation intensities at beta compared to 282 alpha (see Figure $5 \mathrm{~A}$ ) is in line with previous research suggesting that the somatosensory system has 283 a temporal resonance frequency in the beta band (Müller, Neuper, \& Pfurtscheller, 2001; Snyder, 1992; 284 Tobimatsu, Zhang, \& Kato, 1999), thus, responding with greater amplitudes to stimulation frequencies 285 in beta. The same approach was used to detect effects on ITPC across the two frequency bands and 286 three stimulation intensities. Again, we found a main effect of stimulation intensity $(F(2,20)=24.8, p$ $<0.001)$, but not of frequency band $(\mathrm{F}(1,21)=1.0, \mathrm{p}=0.341)$. Post-hoc comparisons between 288 intensities revealed a significant difference between high intensity compared to the other two, but not 289 between sub-threshold and low levels (sub-threshold vs. low: $p=0.293$; sub-threshold vs. high: $p<$ 290 0.001; low vs. high: $\mathrm{p}<0.001)$. Further, no significant interaction between frequency band and 291 stimulation intensity on ITPC was observed $(\mathrm{F}(2,20)=2.7, \mathrm{p}=0.093)$. We conclude that rhythmic 292 vibrotactile stimulation has similar effects on alpha and beta band frequencies regarding phase-angle distributions across trials (ITPC). 

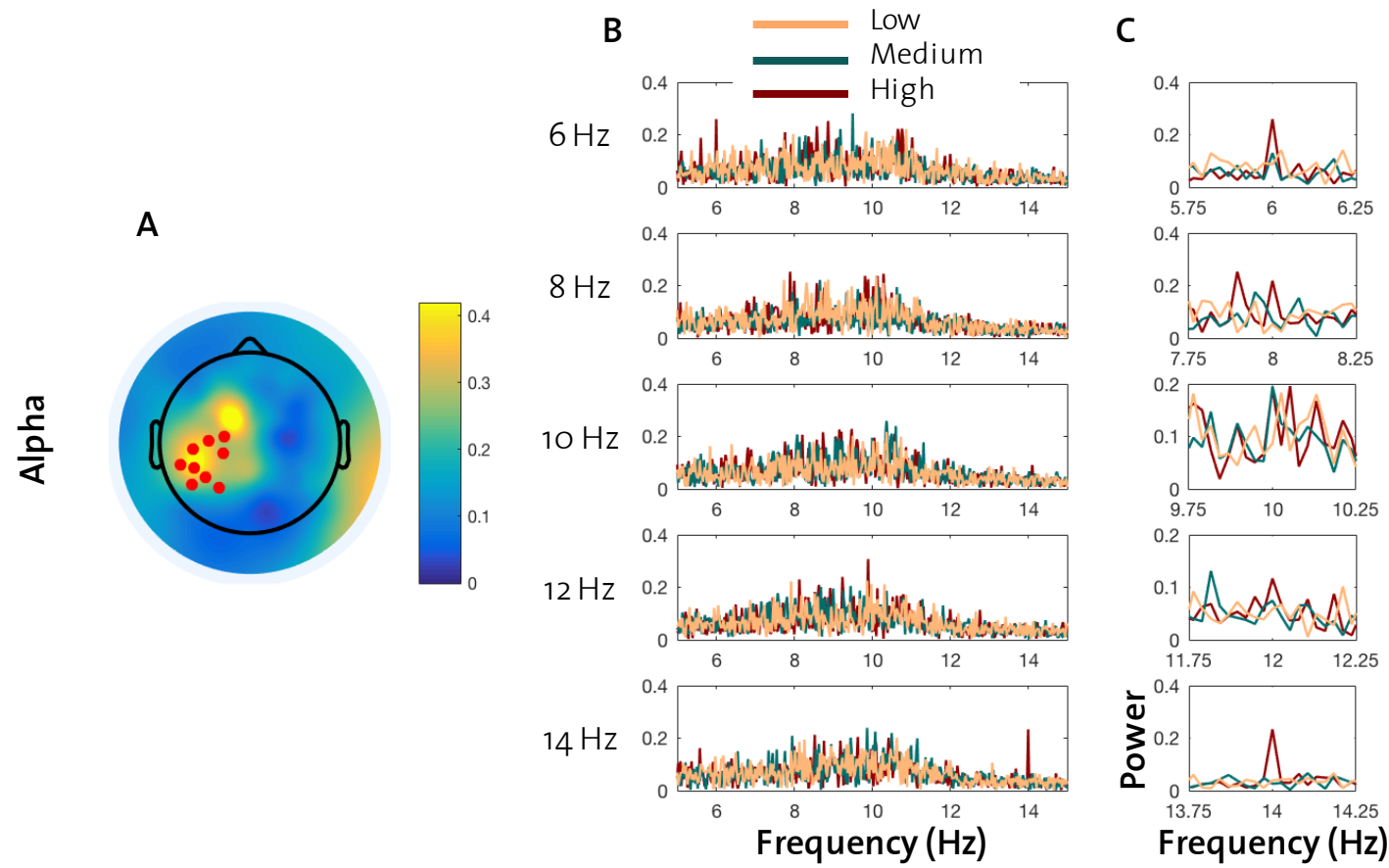

Frequency $(\mathrm{Hz})$
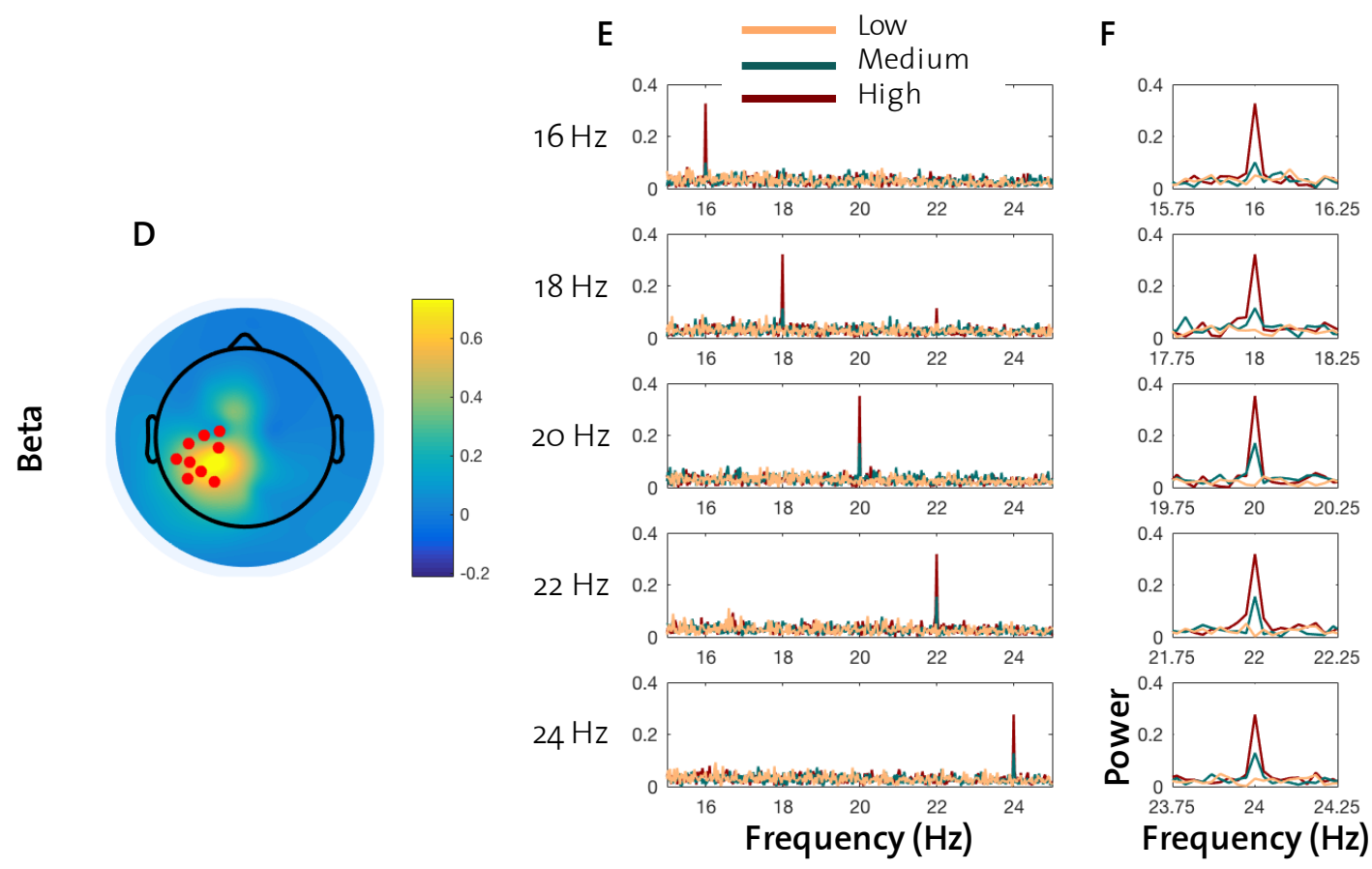

Figure 3. Power-based analysis of each stimulation condition averaged across subjects. A: Topographical plot of changes to electrode power across high-intensity alpha stimulations (red dots: ROI electrodes in the somatosensory area). B: Power spectra (FFT) of time-series of the selected electrode for stimulation frequencies in the alpha range. C: Closer depiction of power spectra at stimulation frequencies. D-F: Equivalent to A-C, across beta stimulation frequencies. 

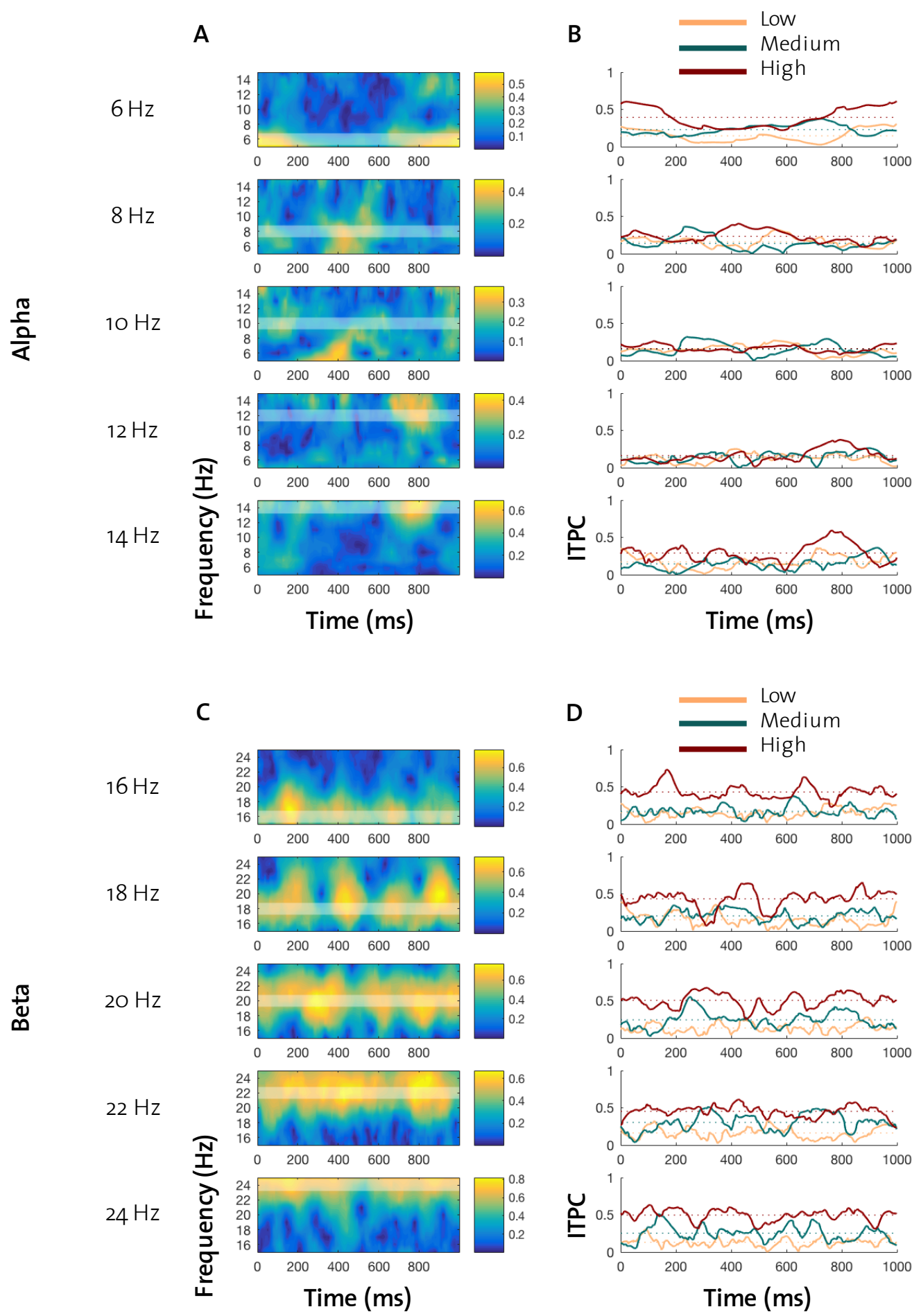

301 Figure 4. Phase-based analysis of each stimulation condition averaged across subjects. Time-series data

302 from chosen electrodes (see Figure 3 A\&D) were cropped in $1 \mathrm{~s}$ segments. A: Time-frequency plots of ITPC for 303 stimulation frequencies delivered with high intensity in the alpha band with indication of the stimulation 304 frequency (white transparent area). B: Time course of ITPC for each stimulation across alpha conditions. Dotted 

trials). C\&D: Equivalent to A\&B, across beta stimulation frequencies.

\section{A}

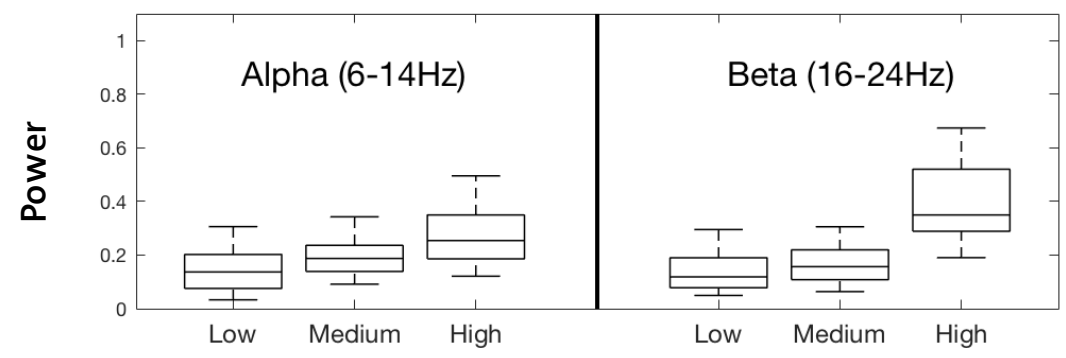

B

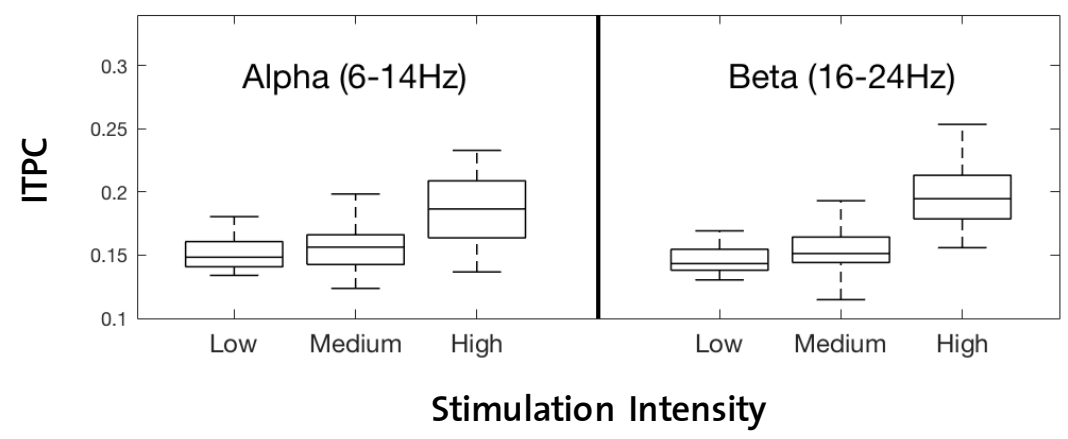

Figure 5. Differences of vibrotactile stimulation on frequency-specific power and ITPC across both main effect of intensity seems to be similar for alpha and beta (no interaction effect of frequency band and intensity). B: Also for ITPC we found a main effect of stimulation intensity. However, a significant interaction effect between frequency band and intensity suggests that the intensity effect differs between alpha and beta.

\section{ISPC in alpha and beta depend on stimulation intensity}

314 To investigate entrainment effects of the stimulation signals, we computed changes to intersite phase

315 clustering ( $\triangle I S P C$ ) for each condition and used linear mixed effects models for the statistical analysis.

316 Our model for the alpha band conditions included stimulation intensity and absolute distance from

317 stimulation frequency to IAF $(\triangle \mathrm{IAF})$ as fixed factors. Any variations in slope and intercept of the data

318 regarding individual variability was accounted for with the inclusion of subject as random variable. To

319 test for an effect of stimulation intensity across all stimulation frequencies, we used mean-shifted $\triangle I A F$

320 values. Using the mean-shifted values allowed us to determine stimulation frequency-independent

321 differences across the three intensity levels by directly comparing the resulting intercepts of the factor

322 stimulation intensity. In line with our results from the power and ITPC analysis, we found the $\triangle I S P C$

323 between stimulation and EEG signal to be more pronounced with increasing stimulation intensity. A 
324 post-hoc Tukey test revealed higher ISPC for the high intensity compared to sub-threshold and low 325 (sub-threshold vs. high: $\mathrm{p}<0.001$; low vs. high: $\mathrm{p}=0.004$ ). However, the increase in $\triangle \mathrm{ISPC}$ from sub-

326 threshold to low intensity showed no difference $(\mathrm{p}=0.936)$. Testing the effect of stimulation intensity

327 in the beta band, revealed similar results as seen for alpha. Again, linear mixed effects models were

328 used with $\triangle \mathrm{ISPC}$ as dependent variable, stimulation intensity and mean-shifted distance from

329 stimulation frequency to IBF $(\triangle \mathrm{IBF})$ as fixed variables and subject as random variable. Positive and

330 statistically significant estimates for the post-hoc comparison between all intensity levels (sub-

331 threshold vs. low: $\mathrm{p}<0.001$; sub-threshold vs. high: $\mathrm{p}<0.001$; low vs. high: $\mathrm{p}<0.001$ ), confirm the

332 effect of stimulation intensity on steady-state neuronal response as hypothesized.

\section{Phase-coupling in beta depends on the interaction of intensity and distance to IBF}

334 In order to test the main requirement regarding entrainment effects, and therefore the most important

335 feature of the Arnold tongue, the dependency of stimulation effects on endogenous neuronal activity

336 was investigated. We started with the analysis of the data obtained from stimulation conditions

337 targeting alpha oscillations (stimulation frequencies from 6 to $14 \mathrm{~Hz}$ ). The shape of the Arnold tongue

338 suggests a dependency of entrainment effects (here: $\triangle$ ISPC) on the distance between stimulation

339 frequency to endogenous peak frequency $(\triangle \mathrm{I} A F / \mathrm{IBF})$. In our statistical model this dependency can be

340 depicted by plotting $\triangle \mathrm{ISPC}$ values according to their $\triangle \mathrm{IAF} / \mathrm{IBF}$ (see Figure $6 \mathrm{~A} / \mathrm{B}$ ). Negative slopes

341 (derived for each stimulation intensity) which significantly differ from 0 can be expected, if the

342 hypothesized dependency is true (i.e. stimulation frequencies closer to IAF/IBF lead to higher $\triangle$ ISPC

343 values). Although the slopes in the alpha conditions for low and high intensities show a negative

344 direction (see Figure $6 \mathrm{~A})$, statistically, they were not significantly different from $0(\Delta \mathrm{IAF}:$ low: $\mathrm{p}=$

345 0.998; $\triangle \mathrm{IAF}$ :high: $\mathrm{p}=0.915$ ). Our next analysis focused on the beta band conditions (stimulation

346 frequencies from 16 to $24 \mathrm{~Hz}$ ). For beta, the slope of the low intensity differed significantly from 0 (p

$347=0.013)$, while sub-threshold and high intensities showed no such effect ( $\triangle \mathrm{IBF}:$ sub-threshold: $\mathrm{p}=$

348 0.998; $\triangle \mathrm{IBF}:$ high: $\mathrm{p}=0.999)$. Our results from the beta band reveal a relationship between endogenous

349 oscillations, stimulation intensity and phase coupling as would be assumed by the Arnold tongue. In

350 our low intensity conditions, the distance of the stimulation frequency to IBF plays a crucial role in

351 predicting coupling between stimulation and the underlying oscillation. The Arnold tongue widens by

352 increasing the stimulation intensity, inferring a decreased effect of $\triangle I B F$. This is because the system

353 experiences such a strong enforcement that the ongoing oscillatory activity has no effect on ISPC. 
A

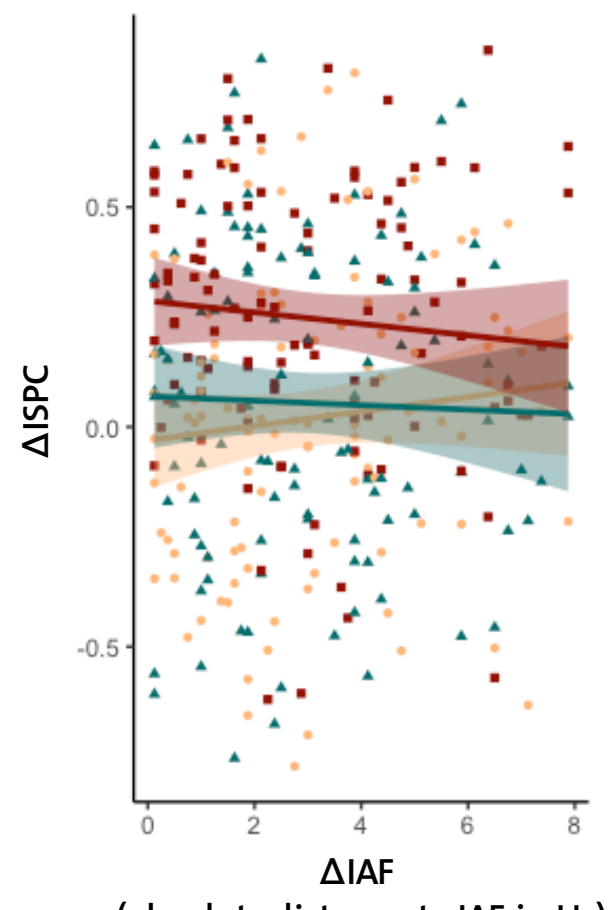

(absolute distance to IAF in $\mathrm{Hz}$ )
B

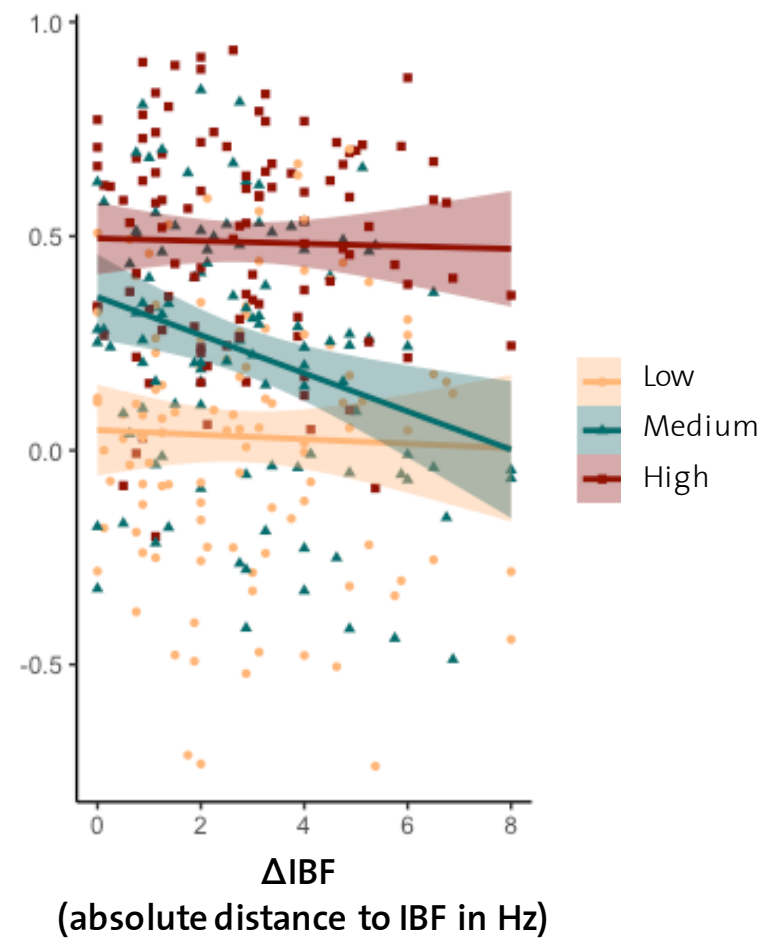

Figure 6. Depiction of the effects of different stimulation intensities on changes in ISPC in relation to the distance to endogenous frequencies (IAF and IBF). A: Stimulation conditions in the alpha band reveal no difference from 0 for any of the slopes and therefore no evidence for entrainment. B: In the beta band, low stimulation intensity reveals a negative slope which differs significantly from 0 . This dependency of entrainment effect ( $\triangle \mathrm{ISPC}$ ) is in line with the hypothesized shape of the Arnold tongue.

\section{Discussion}

362 In the present study, we investigated the effects of rhythmic vibrotactile stimulation across alpha and 363 beta frequencies on neuronal activity in the somatosensory cortex. Our main results reveal a clear effect 364 of stimulation intensity on frequency-specific power and intertrial phase clustering (ITPC) across both

365 frequency bands. A significant interaction effect revealed a difference between alpha and beta 366 stimulation frequencies with regard to their effect on frequency-specific power. The steeper power 367 increase across the three stimulation intensities in beta compared to alpha, as well as the higher power 368 values attained from high-intensity stimulation (Figure $5 \mathrm{~A}$ ) is in line with previous findings suggesting 369 that the resonance frequency of the somatosensory system is in the beta band (Müller et al., 2001; 370 Snyder, 1992; Tobimatsu et al., 1999). Further, we provide evidence for the hypothesized entrainment 
effect by revealing a dependency of phase-locked activity between stimulation signal and endogenous beta brain oscillations on the interaction of stimulation intensity and frequency regarding its distance to individual beta peaks. This dependency is in line with the hypothesized Arnold tongue which

374 predicts that entrainment via external signals of low intensity is stronger when the stimulation

375 frequency matches the frequency of the endogenous system (Fröhlich, 2015; Notbohm et al., 2016;

376 Pikovsky et al., 1999). Stimulation conditions in the alpha band revealed no such entrainment effect although effects of stimulation intensity on ISPC were found as well.

\section{Rhythmic sensory stimulation reveals online entrainment effects}

Entrainment effects of sensory and non-invasive transcranial stimulation have been reported in various studies (for reviews see: Thut et al., 2011; Zoefel et al., 2018), yet our study is the first to demonstrate this effect for a wider range of endogenous brain oscillations in the somatosensory cortex. Our main finding that external rhythmic stimulation interacts with ongoing endogenous brain rhythms of the sensorimotor cortex is in line with previous reports using either sensory stimulation (Gulbinaite, van Viegen, Wieling, Cohen, \& VanRullen, 2017; Notbohm et al., 2016) or various forms of rhythmic, non-invasive brain stimulation methods (Helfrich et al., 2014; Minami \& Amano, 2017; Zaehle et al., 2010). However, until now entrainment effects have been mainly studied by targeting the visual alpha rhythm and its behavioral correlates. Previous studies revealed that steady-state evoked potentials depend on endogenous brain oscillations (Gulbinaite et al., 2017; Notbohm et al., 2016) and that modulating these endogenous rhythms of the visual cortex affects visual processing as measured at the behavioral level (Cecere, Rees, \& Romei, 2015; Ronconi, Busch, \& Melcher, 2018; Ronconi \& Melcher, 2017). Together, these findings provide evidence for alpha entrainment effects in the visual system and also demonstrate the underlying causal relationship between brain oscillations and human behavior.

Few studies have investigated the modulation of neuronal oscillations in human somatosensory cortex. Two recent studies used transcranial alternating current stimulation (tACS) of the mu-rhythm (i.e. the alpha rhythm of sensorimotor cortex) to modulate sensorimotor perception threshold (Gundlach, mu-frequencies (Gundlach, Muller, Nierhaus, Villringer, \& Sehm, 2017). In a first study, Gundlach et al. (2016) applied tACS stimulation at individual mu-alpha frequencies and revealed phase-dependent modulations of human somatosensory perception. However, in a tACS-EEG follow-up study, 
oscillations, their findings reveal a counter-intuitive effect of a decrease of mu-alpha amplitude after stimulation (Gundlach et al., 2017). While the authors argue that homeostatic neuroplastic processes, as well as the electrode placement (bilateral) might have led to the decrease in mu-alpha, the underlying neural mechanism of their first study remains unclear. Even though their approach is well-suited to reveal functional modulations with tACS, it does not allow to investigate online entrainment effects due to large electrical artifacts. Further, due to the lack of different stimulation intensities, as well as variations in stimulation frequencies away from endogenous frequencies, the Arnold tongue cannot be derived from these studies. In addition, only mu-alpha frequency stimulation was applied, preventing conclusions in regard of other frequency bands. Here, we used tactile stimulation across various

411 frequencies covering alpha and beta frequencies, which was provided mechanically and, therefore, did 412 not interfere with the EEG recordings, thus, enabling the analysis of potential entrainment effects

413 during stimulation. Our results suggest that rhythmic activity in somatosensory cortex can be 414 influenced, but that potential entrainment effects were only found in the beta band.

\section{Stronger response to rhythmic beta stimulation in the somatosensory system}

416 Although studies investigating spontaneous (resting-state) EEG activity, found both beta (mu-beta) and alpha (mu-alpha) oscillations in sensorimotor areas (Hillebrand, Barnes, Bosboom, Berendse, \& Stam, 2012; A. Keitel \& Gross, 2016; Ramkumar, Parkkonen, \& Hyvarinen, 2014), the steady-state evoked potentials in the two frequency bands differ clearly (see Figure 3). This finding is in line with previous studies using rhythmic sensory stimulation, revealing that each sensory system responds maximally to a specific stimulation frequency (for a review: Vialatte et al., 2010). Arguably, this represents a mechanism by which each of the sensory systems processes information optimally (Hutcheon \& Yarom, 2000). Such a temporal tuning function appears in the visual system around $10 \mathrm{~Hz}$ (Regan, 1989), in the auditory system around $40 \mathrm{~Hz}$ (Stapells, Makeig, \& Galambos, 1987) and in the somatosensory system around $21 \mathrm{~Hz}$ (Müller et al., 2001; Snyder, 1992; Tobimatsu et al., 1999; Tobimatsu, Zhang, Suga, \& Kato, 2000). Such resonance mechanisms could be responsible for our mixed results for alpha and beta stimulation frequencies.

\section{True entrainment or rhythmically evoked potentials?}

429 Our results are in line with previously stated dependencies of EEG responses to rhythmic stimulation 430 on endogenous brain oscillations. However, it is difficult to draw a final conclusion on whether steady431 state somatosensory evoked potentials (SSSEPs), as produced in our study with vibrotactile 432 stimulation, represent entrained neural oscillations. As Haegens and Zion-Golumbic (2018) point out 
433 in a recent review, the interpretation of neuronal responses to rhythmic stimulation as neural

434 entrainment might lack the fundamental distinction from a rhythmic series of evoked potentials.

435 Although some studies have shown that non-rhythmic (= jittered) stimulations do not reveal a non-

436 linear response with dependency on endogenous oscillations (Kayser, Ince, Gross, \& Kayser, 2015;

437 Notbohm et al., 2016), others found the opposite effect (Capilla et al., 2011; C. Keitel, Thut, \& Gross,

438 2017; see also: Zoefel et al., 2018). Our findings can be seen in agreement with an entrainment

439 mechanism, as we find dependencies of SSSEPs on endogenous oscillations only with a low

440 stimulation intensity and only in beta. Arguably, if SSSEPs would in fact represent only regular

441 repetitions of evoked potentials, we should have seen the same effects for alpha and beta stimulation.

442 However, we do not evaluate our finding in comparison to non-rhythmic stimulation. Regarding the

443 ongoing debate on whether neural entrainment occurs in response to rhythmic sensory stimulation,

444 modulating functional correlates of neural oscillations (e.g. perception, memory) would serve as a

445 convincing outcome measure (Haegens \& Zion Golumbic, 2018). Further, in the present study, we

446 used sensory data from selected electrodes that revealed strongest frequency-specific power changes

447 in a finger tapping task prior to the main experiment. Although our data reveal clear peaks in the alpha

448 and beta bands for most of the participants, our analyses have to be interpreted with caution. For

449 example, a decline in attention and alertness throughout the experiment could have led to variations in

450 IAF and/or IBF (Mierau, Klimesch, \& Lefebvre, 2017), reducing potential entrainment effects as

451 analyzed here. Nevertheless, our findings provide valuable insight in the neural processing of rhythmic

452 sensory stimulation and should be considered in future studies using somatosensory entrainment.

\section{Conclusion}

455 The concept of modulating brain oscillations by rhythmic stimulation has become increasingly popular 456 in research on brain rhythms and their underlying functions (Thut et al., 2011). Despite the limitations 457 of the present study, we show that there is a significant frequency-specific dependency of brain 458 responses to rhythmic vibrotactile stimulation on individual neuronal activity in the beta band. We 459 conclude that our findings support the notion of entrainment of endogenous brain oscillations in 460 somatosensory cortex. This opens up possibilities to causally test interactions between oscillatory 461 activity and behavior by utilizing entrainment via somatosensory stimulation. 


\section{References}

464

465

466

467

468

469

470

471

472

473

474

475

476

477

478

479

480

481

482

483

484

485

486

487

488

489

490

491

492

493

494

495

496

497

498

499

500

501

502

503

Baayen, R. H., Davidson, D. J., \& Bates, D. M. (2008). Mixed-effects modeling with crossed random effects for subjects and items. Journal of Memory and Language, 59(4), 390-412. doi:10.1016/j.jml.2007.12.005

Bates, D., Mächler, M., Bolker, B., \& Walker, S. (2015). Fitting Linear Mixed-Effects Models Usinglme4. Journal of Statistical Software, 67(1). doi:10.18637/jss.v067.i01

Bauer, M., Oostenveld, R., Peeters, M., \& Fries, P. (2006). Tactile spatial attention enhances gammaband activity in somatosensory cortex and reduces low-frequency activity in parieto-occipital areas. J Neurosci, 26(2), 490-501. doi:10.1523/JNEUROSCI.5228-04.2006

Brainard, D. H. (1997). The Psychophysics Toolbox. Spatial Vision, 10(4), 433-436.

Breitwieser, C., Kaiser, V., Neuper, C., \& Müller-Putz, G. R. (2012). Stability and distribution of steady-state somatosensory evoked potentials elicited by vibro-tactile stimulation. Med Biol Eng Comput, 50(4), 347-357. doi:10.1007/s11517-012-0877-9

Capilla, A., Pazo-Alvarez, P., Darriba, A., Campo, P., \& Gross, J. (2011). Steady-state visual evoked potentials can be explained by temporal superposition of transient event-related responses. PLoS One, 6(1), e14543. doi:10.1371/journal.pone.0014543

Cecere, R., Rees, G., \& Romei, V. (2015). Individual differences in alpha frequency drive crossmodal illusory perception. Curr Biol, 25(2), 231-235. doi:10.1016/j.cub.2014.11.034

Cohen, M. X. (2014). Analyzing neural time series data: theory and practice: MIT press.

Crone, N. E., Miglioretti, D. L., Gordon, B., Sieracki, J. M., Wilson, M. T., Uematsu, S., \& Lesser, R. P. (1998). Functional mapping of human sensorimotor cortex with electrocorticographic spectral analysis I. Alpha and beta event-related desynchronization. Brain, 121, 2271-2299.

Delorme, A., \& Makeig, S. (2004). EEGLAB: an open source toolbox for analysis of single-trial EEG dynamics including independent component analysis. J Neurosci Methods, 134(1), 9-21. doi:10.1016/j.jneumeth.2003.10.009

Fransen, A. M., Dimitriadis, G., van Ede, F., \& Maris, E. (2016). Distinct alpha- and beta-band rhythms over rat somatosensory cortex with similar properties as in humans. $J$ Neurophysiol, 115(6), 3030-3044. doi:10.1152/jn.00507.2015

Fröhlich, F. (2015). Experiments and models of cortical oscillations as a target for noninvasive brain stimulation. Progress in Brain Research, 222, 41-73. doi:10.1016/bs.pbr.2015.07.025

Gulbinaite, R., van Viegen, T., Wieling, M., Cohen, M. X., \& VanRullen, R. (2017). Individual Alpha Peak Frequency Predicts $10 \mathrm{~Hz}$ Flicker Effects on Selective Attention. J Neurosci, 37(42), 10173-10184. doi:10.1523/JNEUROSCI.1163-17.2017

Gundlach, C., Muller, M. M., Nierhaus, T., Villringer, A., \& Sehm, B. (2017). Modulation of Somatosensory Alpha Rhythm by Transcranial Alternating Current Stimulation at MuFrequency. Front Hum Neurosci, 11, 432. doi:10.3389/fnhum.2017.00432

Gundlach, C., Müller, M. M., Nierhaus, T., Villringer, A., \& Sehm, B. (2016). Phasic Modulation of Human Somatosensory Perception by Transcranially Applied Oscillating Currents. Brain Stimulation, 9(5), 712-719. doi:10.1016/j.brs.2016.04.014

Haegens, S., \& Zion Golumbic, E. (2018). Rhythmic facilitation of sensory processing: A critical review. Neurosci Biobehav Rev, 86, 150-165. doi:10.1016/j.neubiorev.2017.12.002 
504

505

506

507

508

509

510

511

512

513

514

515

516

517

518

519

520

521

522

523

524

525

526

527

528

529

530

531

532

533

534

535

536

537

538

539

540

541

542

543

544

545

Helfrich, R. F., Schneider, T. R., Rach, S., Trautmann-Lengsfeld, S. A., Engel, A. K., \& Herrmann, C. S. (2014). Entrainment of brain oscillations by transcranial alternating current stimulation. Curr Biol, 24(3), 333-339. doi:10.1016/j.cub.2013.12.041

Hillebrand, A., Barnes, G. R., Bosboom, J. L., Berendse, H. W., \& Stam, C. J. (2012). Frequencydependent functional connectivity within resting-state networks: an atlas-based MEG beamformer solution. Neuroimage, 59(4), 3909-3921. doi:10.1016/j.neuroimage.2011.11.005

Hutcheon, B., \& Yarom, Y. (2000). Resonance, oscillation and the intrinsic frequency preferences of neurons. Trends in Neuroscience, 23, 216-222.

Johansson, R. S., \& Flanagan, J. R. (2009). Coding and use of tactile signals from the fingertips in object manipulation tasks. Nat Rev Neurosci, 10(5), 345-359. doi:10.1038/nrn2621

Kayser, S. J., Ince, R. A., Gross, J., \& Kayser, C. (2015). Irregular Speech Rate Dissociates Auditory Cortical Entrainment, Evoked Responses, and Frontal Alpha. J Neurosci, 35(44), 14691-14701. doi:10.1523/JNEUROSCI.2243-15.2015

Keitel, A., \& Gross, J. (2016). Individual Human Brain Areas Can Be Identified from Their Characteristic Spectral Activation Fingerprints. PLoS Biol, 14(6), e1002498. doi:10.1371/journal.pbio. 1002498

Keitel, C., Quigley, C., \& Ruhnau, P. (2014). Stimulus-driven brain oscillations in the alpha range: entrainment of intrinsic rhythms or frequency-following response? J Neurosci, 34(31), 1013710140. doi:10.1523/JNEUROSCI.1904-14.2014

Keitel, C., Thut, G., \& Gross, J. (2017). Visual cortex responses reflect temporal structure of continuous quasi-rhythmic sensory stimulation. Neuroimage, 146, 58-70. doi:10.1016/j.neuroimage.2016.11.043

Kilavik, B. E., Zaepffel, M., Brovelli, A., MacKay, W. A., \& Riehle, A. (2013). The ups and downs of beta oscillations in sensorimotor cortex. Exp Neurol, 245, 15-26. doi:10.1016/j.expneurol.2012.09.014

Liu, Q., Ganzetti, M., Wenderoth, N., \& Mantini, D. (2018). Detecting Large-Scale Brain Networks Using EEG: Impact of Electrode Density, Head Modeling and Source Localization. Front Neuroinform, 12, 4. doi:10.3389/fninf.2018.00004

Mierau, A., Klimesch, W., \& Lefebvre, J. (2017). State-dependent alpha peak frequency shifts: Experimental evidence, potential mechanisms and functional implications. Neuroscience, 360, 146-154. doi:10.1016/j.neuroscience.2017.07.037

Minami, S., \& Amano, K. (2017). Illusory Jitter Perceived at the Frequency of Alpha Oscillations. Curr Biol, 27(15), 2344-2351 e2344. doi:10.1016/j.cub.2017.06.033

Müller, G. R., Neuper, C., \& Pfurtscheller, G. (2001). „Resonance-like“ frequencies of sensorimotor areas evoked by repetitive tactile stimulation. Biomedical Engineering, 46(7-8), 186-190.

Notbohm, A., Kurths, J., \& Herrmann, C. S. (2016). Modification of Brain Oscillations via Rhythmic Light Stimulation Provides Evidence for Entrainment but Not for Superposition of EventRelated Responses. Front Hum Neurosci, 10, 10. doi:10.3389/fnhum.2016.00010

Oldfield, R. C. (1971). The Assessment and Analysis of Handedness: The Edinburgh Inventory. Neuropsychologia, 9, 97-113.

Pfurtscheller, G., \& Lopes da Silva, F. H. (1999). Event-related EEG/MEG synchronization and desynchronization: basic principles. Clinical Neurophysiology, 110, 1842-1857. 
Pikovsky, A. S., Rosenblum, M. G., Zaks, M. A., \& Kurths, J. (1999). Phase Synchronization of Regular and Chaotic oscillators. In H. G. Schuster (Ed.), Handbook of Chaos Control. Weinheim: Wiley-VCH.

Ploner, M., Gross, J., Timmermann, L., Pollok, B., \& Schnitzler, A. (2006). Oscillatory activity reflects the excitability of the human somatosensory system. Neuroimage, 32(3), 1231-1236. doi:10.1016/j.neuroimage.2006.06.004

R Core Team. (2018). R: A language and environment for statistical computing. Vienna, Austria. Retrieved from https://www.r-project.org/

Rajagovindan, R., \& Ding, M. (2011). From Prestimulus Alpha Oscillation to Visual-evoked Response: An Inverted-U Function and Its Attentional Modulation. Journal of Cognitive Neuroscience, 23(6), 1379-1394.

Ramkumar, P., Parkkonen, L., \& Hyvarinen, A. (2014). Group-level spatial independent component analysis of Fourier envelopes of resting-state MEG data. Neuroimage, 86, 480-491. doi:10.1016/j.neuroimage.2013.10.032

Regan, D. (1977). Steady-state evoked potentials. JOSA, 67(11), 1475-1489.

Regan, D. (1989). Human brain electrophysiology: evoked poten-tials and evoked magnetic fields in science and medicine. New York: Elsevier.

Ronconi, L., Busch, N. A., \& Melcher, D. (2018). Alpha-band sensory entrainment alters the duration of temporal windows in visual perception. Sci Rep, 8(1), 11810. doi:10.1038/s41598-01829671-5

Ronconi, L., \& Melcher, D. (2017). The Role of Oscillatory Phase in Determining the Temporal Organization of Perception: Evidence from Sensory Entrainment. J Neurosci, 37(44), 1063610644. doi:10.1523/JNEUROSCI.1704-17.2017

Schwab, K., Ligges, C., Jungmann, T., Hilgenfeld, B., Haueisen, J., \& Witte, H. (2006). Alpha entrainment in human electroencephalogram and magnetoencephalogram recordings. Neuroreport, 17(17), 1829-1833.

Snyder, A. Z. (1992). Steady-state vibration evoked potentials: description of technique and characterization of responses. Electroencephalography and Clinical Neurophysiology/Evoked potentials Section, 84(3), 257-268.

Spitzer, B., \& Haegens, S. (2017). Beyond the Status Quo: A Role for Beta Oscillations in Endogenous Content (Re)Activation. eNeuro, 4(4). doi:10.1523/ENEURO.0170-17.2017

Stapells, D. R., Makeig, S., \& Galambos, R. (1987). Auditory steady-state responses: threshold prediction using phase coherence. Electroencephalography and Clinical Neurophysiology, 67(3), 260-270.

Thut, G., Schyns, P. G., \& Gross, J. (2011). Entrainment of perceptually relevant brain oscillations by non-invasive rhythmic stimulation of the human brain. Front Psychol, 2, 170. doi:10.3389/fpsyg.2011.00170

Tobimatsu, S., Zhang, Y. M., \& Kato, M. (1999). Steady-state vibration somatosensory evoked potentials: physiological characteristics and tuning function. Clinical Neurophysiology, 110(11), 1953-1958.

Tobimatsu, S., Zhang, Y. M., Suga, R., \& Kato, M. (2000). Differential temporal coding of the vibratory sense in the hand and foot in man. Clinical Neurophysiology, 111(3), 398-404. 
588 Vialatte, F. B., Maurice, M., Dauwels, J., \& Cichocki, A. (2010). Steady-state visually evoked potentials: focus on essential paradigms and future perspectives. Prog Neurobiol, 90(4), 418438. doi:10.1016/j.pneurobio.2009.11.005

Zaehle, T., Rach, S., \& Herrmann, C. S. (2010). Transcranial alternating current stimulation enhances individual alpha activity in human EEG. PLoS One, 5(11), e13766. doi:10.1371/journal.pone.0013766

594 Zoefel, B., Ten Oever, S., \& Sack, A. T. (2018). The Involvement of Endogenous Neural Oscillations in the Processing of Rhythmic Input: More Than a Regular Repetition of Evoked Neural Responses. Front Neurosci, 12, 95. doi:10.3389/fnins.2018.00095 